\title{
Soins virtuels pour les patients atteints de la maladie d'Alzheimer et de démences connexes à l'ère de la COVID-19 et au-delà
}

\author{
Paula J. Gosse BSc, Charles D. Kassardjian MD MSc, Mario Masellis MD PhD, Sara B. Mitchell MD MSP
}

Citation : CMAJ 2021 March 15;193:E371-7. doi : 10.1503/cmaj.201938-f

Voir la version anglaise de l'article ici : www.cmaj.ca/lookup/doi/10.1503/cmaj.201938

$\mathbf{P}$

endant la pandémie de maladie à coronavirus 2019 (COVID-19), l'Association médicale canadienne et les autorités sanitaires provinciales ont conseillé aux médecins d'offrir des soins virtuels à leurs patients lorsque c'est possible. Les soins virtuels, ou la télémédecine, se définissent comme " toute interaction entre patients, entre personnes qui participent à leurs soins ou entre membres de ces deux groupes ayant lieu à distance, utilisant une forme de technologie de l'information ou des communications et visant à améliorer ou à optimiser la qualité et l'efficacité des soins aux patients ${ }^{1}$ ». Ce mode de soins est de plus en plus utilisé pour surmonter les obstacles physiques à la prestation des soins afin, entre autres, d'en améliorer la disponibilité et l'accessibilité dans les régions rurales et éloignées². Plus récemment, les mesures de distanciation physique rendues nécessaires par la pandémie sont devenues une raison impérative d'intégrer les soins virtuels aux infrastructures sanitaires existantes.

Les personnes atteintes de la maladie d'Alzheimer et de démences connexes (encadré 1 ) peuvent éprouver des difficultés particulières à accéder aux soins de santé qu'elles requièrent, par exemple, difficultés à reconnaître et à exprimer leurs besoins en matière de soins et à s'y retrouver dans les méandres du système de santé ${ }^{7,8}$. La pandémie de COVID-19

\section{Encadré 1: Maladie d'Alzheimer et démences} connexes

L'atteinte cognitive couvre un large spectre qui va de l'atteinte cognitive légère ${ }^{3}$ à la démence, selon la gravité des déficits et leurs effets sur le fonctionnement quotidien. La maladie d'Alzheimer et les démences connexes forment un groupe de maladies neurodégénératives caractérisées par une perte cognitive progressive qui affecte le fonctionnement quotidien. La maladie d'Alzheimer, avec ou sans maladie vasculaire cérébrale, est la plus fréquente cause de démence ${ }^{4}$; les autres causes de démence incluent la démence à corps de Lewy ${ }^{5}$ et la dégénérescence lobaire frontotemporale ${ }^{6}$.

\section{POINTS CLÉS}

- Les soins virtuels recèlent le potentiel pour améliorer l'accès aux soins de santé des personnes atteintes de la maladie d'Alzheimer et de démences connexes, surtout dans le contexte de la pandémie de maladie à coronavirus 2019 (COVID-19).

- La consultation par visioconférence est une approche bien étudiée pour la prestation des soins virtuels; elle a été utilisée avec succès pour évaluer, diagnostiquer et prendre en charge à distance cette population de patients.

- Les programmes de soins virtuels qui fonctionnent ont souvent accès à des renseignements cliniques supplémentaires pour aider au diagnostic et mettent à contribution des équipes interdisciplinaires formées pour gérer la complexité des cas.

- Les données sont insuffisantes en ce qui concerne les consultations virtuelles directes à domicile pour les personnes atteintes de la maladie d'Alzheimer et de démences connexes; ce modèle est associé à des difficultés particulières qui devront entrer en ligne de compte à mesure que les soins virtuels se répandront comme façon de prodiguer des soins.

peut aggraver ces difficultés, car les patients qui se fient habituellement à des personnes pour vaquer à leurs occupations quotidiennes, telles qu'assurer leur transport ou les accompagner à leurs rendez-vous médicaux sont affectés de manière disproportionnée par les mesures de distanciation physique 9 . Tout changement à la routine ou aux formes d'aide habituelles peut aussi aggraver les symptômes neuropsychiatriques des patients, et le risque d'épuisement chez les proches aidants $^{10-12}$. En outre, une évaluation de 2016 estimait que le nombre de personnes atteintes de démence allait presque doubler en 15 ans $^{13}$, ce qui signifie qu'il faut de nouveaux modèles efficaces et économiques de prestation des soins pour cette population dans les meilleurs délais.

Les soins virtuels sont prometteurs pour ce qui est de surmonter plusieurs de ces difficultés tout en permettant aux patients de recevoir leurs soins en toute sécurité, à la maison ou au centre de soins de longue durée ${ }^{14}$. Mais le déploiement 
Encadré 2 : Données utilisées pour la présente revue

Nous avons interrogé les réseaux MEDLINE, Embase et PubMed pour recenser les articles pertinents publiés entre le

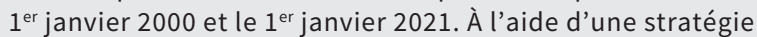
portant sur des mots clés relatifs aux soins virtuels et à la démence, nous avons inclus tous les articles portant sur le recours aux soins virtuels pour une consultation clinique dans le but d'évaluer ou de prendre en charge l'atteinte cognitive légère, la maladie d'Alzheimer ou une démence connexe, mais non sur la téléréadaptation, les applications et appareils mobiles ou le soutien virtuel aux proches aidants. Nous avons passé en revue la documentation, organisé les données en catégories thématiques et synthétisé les résultats (voir annexe 1 , accessible en anglais au www.cmaj.ca/lookup/doi/10.1503/cmaj.201938/tab -related-content pour plus de détails sur la méthodologie). Compte tenu du nombre limité d'essais cliniques randomisés dans ce domaine, la majeure partie des données probantes de la présente revue provient d'études test-retest, d'études d'observation, et d'évaluations rétrospectives de programmes de soins virtuels. Les données probantes utilisées pour les recommandations au tableau 2 proviennent principalement de l'opinion d'experts et des avis issus d'études et de programmes de soins virtuels antérieurs.

rapide et à grande échelle des soins virtuels doit se faire avec prudence. Nous passons en revue les publications touchant un large éventail de spécialités, dont la neurologie, la gériatrie et la psychiatrie, pour faire une synthèse des données probantes et des recommandations à l'intention des généralistes et des spécialistes qui adoptent une plateforme virtuelle pour soigner leurs patients atteints de démence, en tenant compte des obstacles potentiels à son adoption (encadré 2).

\section{Les consultations virtuelles conviennent- elles pour l'évaluation et le diagnostic des démences?}

L'évaluation diagnostique d'un patient chez qui on soupçonne une possible maladie d'Alzheimer ou une démence connexe repose sur une anamnèse complète, la vérification de ses capacités fonctionnelles, une entrevue d'information avec un tiers, des examens cognitifs et neurologiques, en plus d'une revue des analyses pertinentes. Certains aspects du processus diagnostique cadrent mieux que d'autres avec la plateforme virtuelle. Le diagnostic clinique des démences repose sur une évaluation par des experts ${ }^{3,4}$, et selon des groupes de travail en téléneurologie, les démences pourraient se prêter à une évaluation à distance ${ }^{15}$.

\section{Consultation par téléphone}

Des outils d'évaluation cognitive par téléphone ont été utilisés dans des contextes épidémiologiques et cliniques ${ }^{16}$. L'entrevue téléphonique sur l'état cognitif (ou TICS, pour Telephone Interview for Cognitive Status), un instrument populaire inspiré du mini-examen de l'état mental (MEEM), est dotée d'excellentes sensibilité $(94 \%)$ et spécificité (100\%) pour le dépistage de la démence, présentant une bonne corrélation entre les scores du MEEM et de la TICS ${ }^{17}$. La TIC modifiée (TICS-M) peut être utile pour distinguer l'atteinte cognitive légère de la fonction cognitive normale parce qu'elle évalue le rappel verbal différé ${ }^{18,19}$, même si sa capacité de situer les cas dans tout le spectre de l'atteinte cognitive est limité ${ }^{19}$. D'autres outils validés de dépistage de l'atteinte cognitive incluent des MEEM adaptés pour le téléphone et le t-MOCA (telephone-based Montreal Cognitive Assessment) ${ }^{16,20}$, même si ce dernier n'a été validé que chez les patients atteints de maladie vasculaire cérébrale $e^{21}$. Donc, même si l'approche téléphonique a été suggérée comme méthode efficace d'évaluation cognitive ${ }^{16,22}$, elle comporte des limites, telles que la difficulté d'évaluer l'atteinte visuospatiale et la capacité de nommer des objets. Finalement, pour un nouveau diagnostic clinique de la maladie d'Alzheimer ou d'une démence connexe, la consultation téléphonique risque de ne pas générer suffisamment de renseignements ${ }^{16,22}$.

\section{Consultation par visioconférence}

La consultation par visioconférence (CVC) est probablement le seul mode de télémédecine susceptible de pouvoir remplacer les examens en personne lors d'un nouveau diagnostic de la maladie d'Alzheimer ou d'une démence connexe $\mathrm{e}^{23}$. Une métaanalyse de 2017 a constaté que les scores aux tests neuropsychologiques obtenus par CVC sont comparables à ceux qu'on obtient en personne, même si on a noté plus de discordances quand les connexions Internet sont plus lentes et dans les cohortes plus âgées ${ }^{24}$. Une récente revue systématique a quant à elle révélé que l'atteinte cognitive et l'utilisation de méthodes d'évaluation non traditionnelles, y compris dans un environnement domestique et sans supervision, posaient des difficultés potentielles pour la validité des évaluations à distance de la fonction cognitive ${ }^{25}$. Même si les études individuelles sur les tests cognitifs montrent souvent une bonne fiabilité globale entre les évaluations en personne et par $\mathrm{CVC}^{26-36}$, leur généralisation est limitée car elles excluent souvent les patients qui ont de graves problèmes auditifs, visuels

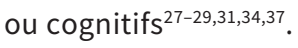

Une étude longitudinale rigoureuse a fait état de différences entre les scores obtenus par CVC et en personne seulement dans les cas d'atteinte cognitive grave. Les scores de ces derniers ont été moins bons à l'évaluation par CVC qu'en personne, ce qui laisse entendre que la prestation des soins virtuels favoriserait une surestimation des déficits cognitifs graves ${ }^{37}$. Le tableau 1 résume les données existantes qui comparent la CVC aux tests d'évaluation cognitive courants administrés en personne chez les patients ayant une atteinte cognitive. D'autres outils, comme l'échelle de dépression gériatrique (EDG) ${ }^{26}$ et l'évaluation des activités de la vie quotidienne, ont aussi été administrés par CVC avec fiabilité.

La consultation par visioconférence s'est révélée utile pour établir à distance un nouveau diagnostic clinique de démence $28,40,41$. Dans une petite étude de cohorte sur des patients présentant une atteinte cognitive non diagnostiquée, la précision de l'évaluation virtuelle de la démence a été mesurée en comparant les diagnostics posés en personne et 
Tableau 1 : Sommaire des études existantes comparant l'administration de tests de fonction cognitive courants lors de consultations par visioconférence c. en personne chez des patients ayant une atteinte cognitive

\begin{tabular}{|c|c|c|c|}
\hline Essai & $\begin{array}{c}N^{\text {bre }} \\
\text { d'études }\end{array}$ & Types de populations étudiées & $\mathrm{CCl}$ disponibles \\
\hline Mini-examen de l'état mental (MEEM) ${ }^{28-31,33,37-39}$ & 8 & Témoins en bonne santé, $\mathrm{ACL}, \mathrm{MA}, \mathrm{DV}$, autre & $0,88-0,92$ \\
\hline Montreal Cognitive Assessment (MOCA) $)^{26,27,32}$ & 3 & Témoins en bonne santé, $\mathrm{ACL}, \mathrm{MA}, \mathrm{DCL}$ & $0,85-0,98$ \\
\hline Boston Naming Test (BNT) $)^{29-31,34,39}$ & 5 & Témoins en bonne santé, $A C L, M A$ & $0,81-0,93$ \\
\hline Test de l'horloge $\mathrm{e}^{29-31,39}$ & 4 & Témoins en bonne santé, $A C L, M A$ & $0,65-0,71$ \\
\hline Test d’apprentissage verbal de Hopkins - révisé (HVLT-R) ${ }^{29-31,39}$ & 4 & Témoins en bonne santé, $A C L, M A$ & $0,54-0,90$ \\
\hline Échelle d'évaluation cognitive pour la MA (ADAS-cog) ${ }^{36,37}$ & 2 & Témoins en bonne santé, $A C L, M A$ & 0,86 \\
\hline
\end{tabular}

par CVC. On a noté une excellente concordance entre les 2 modes diagnostiques ${ }^{40}$. Des études subséquentes sont arrivées à des conclusions similaires ${ }^{28,41}$. Par contre, dans ces études, les médecins avaient souvent accès à des renseignements supplémentaires, comme les résultats de tests neuropsychologiques ${ }^{41}$, d'évaluations fonctionnelles ${ }^{40}$ ou d'examens physiques ${ }^{40}$ préalablement réalisés en personne. Il est donc important de reconnaître les risques et les limites du diagnostic à distance posé sur la base de tests cognitifs et de critères cliniques originalement conçus pour des consultations en personne.

Des programmes de soins virtuels ont montré qu'il est possible d'intégrer la CVC pour améliorer l'accès et le diagnostic des patients atteints de la maladie d'Alzheimer et de démences connexes qui vivent dans des communautés rurales ${ }^{42-46}$. Dans ces programmes, les patients se rendaient à une clinique de soins virtuels près de chez eux pour avoir accès par CVC à un spécialiste d'un grand établissement de santé. Les programmes qui ont eu du succès reposaient sur des équipes interdisciplinaires capables de gérer la complexité des cas, les comorbidités médicales et psychiatriques et les besoins psychosociaux ${ }^{43-45}$. Étant donné qu'il faut pouvoir continuer de diagnostiquer la maladie d'Alzheimer et les démences connexes en temps de pandémie de COVID-19, des guides de pratique clinique ont récemment été préparés pour le déploiement de cliniques de la mémoire virtuelles ${ }^{47}$.

\section{Les consultations virtuelles conviennent-elles pour le suivi et la prise en charge?}

La nature évolutive de la maladie d'Alzheimer et des démences connexes nécessite un suivi et une prise en charge continus des patients. D'autres obstacles à l'accès aux soins en personne peuvent se dresser à mesure que la maladie progresse, par exemple, déclin de la mobilité, désorientation croissante à la moindre modification de la routine habituelle, aggravation des symptômes neuropsychiatriques et dépendance accrue vis-à-vis des proches aidants. Les soins virtuels pourraient minimiser le dérangement occasionné par les consultations en personne chez cette population formée en majeure partie d'adultes âgés et fragiles.

Chez les personnes atteintes de démence, les programmes de soins virtuels ont montré la faisabilité des CVC pour commencer et gérer la prise des médicaments ${ }^{44,48-51}$, orienter les patients et les familles vers les services de soutien ${ }^{42,48,51}$, discuter d'enjeux de sécurité et de planification ${ }^{44,49,51}$, déterminer si d'autres analyses de laboratoire, épreuves d'imagerie ou tests neuropsychologiques sont requis ${ }^{42,48}$ et réévaluer les signes de déclin cognitif ${ }^{46,49}$. Par exemple, après avoir utilisé la CVC pour la prise en charge de leurs patients pendant 5 ans, les professionnels de la santé d'une clinique de la mémoire ont pu identifier ceux dont l'atteinte cognitive légère évoluait vers la démence ${ }^{46}$.

On ignore encore si les soins virtuels ont une influence sur les résultats cliniques; par contre, les études existantes révèlent d'autres avantages de cette approche. Un essai randomisé regroupant 1560 patients atteints de démence et leurs proches aidants a révélé que des consultations de suivi mensuelles par téléphone avec des équipes interdisciplinaires amélioraient la qualité de vie après 12 mois, comparativement aux soins usuels ${ }^{52}$. Dans une étude de cohorte prospective sur des patients atteints de démence, les changements des scores aux MEEM annuels entre les patients suivis par CVC et en personne ont généralement été similaires ${ }^{53}$. Ceux dont l'atteinte était plus bénigne dans le groupe suivi en contexte de soins virtuels ont présenté un déclin moins rapide à terme de leurs scores au MEEM comparativement au groupe suivi en personne, ce qui suggère que les patients ayant un déficit cognitif plus léger pourraient être plus sensibles aux bienfaits des soins virtuels ${ }^{53}$, tels que l'accès plus facile à des soins spécialisés ou à des consultations plus fréquentes. Les patients suivis par CVC ont aussi adhéré à leur traitement pharmacologique plus longtemps que les patients suivis en personne ${ }^{54}$. Finalement, la CVC a été utilisée pour améliorer les symptômes comportementaux et réduire les taux d'hospitalisation chez les patients atteints de démence vivant dans des établissements de soins de longue dureée $e^{55,56}$. 


\section{Que pensent les patients des soins virtuels pour la maladie d'Alzheimer et les démences connexes?}

Pour assurer la viabilité des modèles de soins virtuels, il faut que les patients acceptent les soins virtuels et en soient satisfaits. Les adultes âgés atteints de ces démences semblent accepter les soins virtuels pour les évaluations cognitives; le taux de satisfaction à l'endroit des CVC est élevé ${ }^{37,40,43,44,57}$. Les patients, les proches aidants et les médecins ont aussi exprimé de forts taux de satisfaction à l'endroit des CVC utilisées pour les soins de suivi ${ }^{48,49,58}$. Les préférences quant aux modes de prestation des soins chez les patients varient de préférence à l'endroit des CVC au détriment des consultations en personne $\mathrm{e}^{37,40}$, à absence apparente de préférence ${ }^{57}$. Les avantages des soins virtuels identifiés incluent la commodité et les gains de ressources et de temps, ainsi que l'accès amélioré aux soins spécialisés ${ }^{40,42-45}$. Calculs précis à l'appui, le déploiement des soins virtuels a généré des économies significatives sur les plans des coûts, du temps et du kilométrage ${ }^{48}$. La plupart des consultations virtuelles décrites dans les programmes de télémédecine s'effectuent avec succès, en dépit des obstacles identifiés quant à la satisfaction des usagers, obstacles qui ont souvent trait à des difficultés techniques (qualité du son ou de l'image)27,40,42.

\section{Les soins virtuels permettent-ils d'évaluer et de prendre en charge les patients à la maison?}

Les soins virtuels semblent prometteurs chez cette population de patients, mais à ce jour, la recherche sur les soins virtuels a surtout porté sur la CVC entre 2 établissements de santé. Ce modèle a l'avantage de disposer d'un personnel dûment formé sur place pour faciliter les consultations, en plus de se faire dans des conditions d'évaluation standardisées ${ }^{59}$. Il sera important de vérifier si les soins virtuels directs au patient à son domicile sont aussi prometteurs, particulièrement dans le contexte de la pandémie actuelle, qui impose un confinement pour des raisons de santé et de sécurité.

Peu d'études ont porté sur les CVC directes à domicile pour les patients atteints de la maladie d'Alzheimer et de démences connexes ${ }^{26,60}$. Dans une étude, les évaluations de la démence ont été faisables et fiables chez les patients à domicile, mais la participation des proches aidants s'est révélée essentielle à la réussite des consultations ${ }^{26}$. Une autre étude récente a constaté que si on leur laissait le choix, la plupart des familles déclinaient l'offre de CVC à domicile pour le suivi de la démence en raison des difficultés d'accès à la technologie appropriée, au manque de littératie numérique et à l'absence de soutien à la maison pour faciliter la consultation. Ceux qui ont accepté les CVC à domicile s'en sont déclarés aussi satisfaits que des rencontres en personne ${ }^{60}$. Plusieurs cliniques ont aussi décrit leurs expériences du passage des soins en personne au modèle de soins par CVC directe à domicile chez les patients ayant une atteinte cognitive durant la pandémie $e^{51,61}$. Étant donné le manque de données probantes de qualité au sujet de l'approche par CVC directe à domicile, il faudra en évaluer l'utilisation plus attentivement chez cette population de patients.

\section{Quels sont les principaux obstacles aux soins virtuels directs à domicile pour les patients atteints de démence?}

L'interface virtuelle des CVC directes à domicile pour la démence comporte des limites. Les obstacles aux soins virtuels ont notamment trait à des enjeux technologiques, à la qualité du lien thérapeutique médecin-patient, au processus diagnostique et à la pandémie de COVID-19 (voir tableau 2 pour une liste des obstacles aux soins virtuels et les recommandations associées).

La majorité des patients atteints de la maladie d'Alzheimer et de démences connexes sont des adultes âgés, plus susceptibles d'éprouver des difficultés face aux technologies numériques et aux services Internet requis pour réaliser des consultations virtuelles ${ }^{62,74}$. Le problème est plus marqué dans les régions rurales ou éloignées qui ne disposent pas des ressources nécessaires pour déployer des services de soins virtuels ${ }^{2}$. Outre les problèmes d'accès matériels, certaines personnes n’ont ni la littératie numérique nécessaire et ni la confiance en leur capacité d'utiliser l'ordinateur, l'Internet ou les plateformes de CVC, difficultés que viennent exacerber les atteintes sensorielles qui croissent avec l'âge $\mathrm{e}^{70,75,76}$. Selon une volumineuse étude transversale américaine, $38 \%$ et $20 \%$ des adultes de 65 ans et plus ne se sentaient pas prêts pour la télémédecine par CVC et par téléphone, respectivement ${ }^{74}$. Les préférences des patients doivent entrer en ligne de compte lors du choix d'un mode de soins virtuels, et au besoin, il est possible d'utiliser le téléphone seul ou avec la CVC ${ }^{14,63,64}$. De plus, la présence d'une tierce partie, un proche aidant, par exemple, pour faciliter la consultation virtuelle est essentielle à la réussite des soins virtuels directs à domicile pour la démence et doit être envisagée lorsque les professionnels de la santé offrent de tels soins à cette population ${ }^{26,64,70}$.

Étant donné qu'elles reposent sur la transmission numérique et qu'elles se déroulent dans des environnements domestiques non régulés, les consultations virtuelles sont plus propices à la fuite de données. Les plateformes de soins virtuels doivent être sécurisées et proposer uniquement des soins de santé n'incluant aucune collecte de renseignements personnels, et malgré cela, des fuites de données peuvent se produire. À chaque consultation, il faut obtenir le consentement éclairé du patient ou de son mandataire relativement aux risques liés aux soins virtuels. Il faut aussi vérifier l'identité des patients à chaque consultation et leur demander de choisir un endroit discret pour la consultation ${ }^{14,64}$.

L'ajout d'un examen neurologique ciblé au cours de la consultation virtuelle pour évaluer la maladie d'Alzheimer ou une démence connexe n'a pas fait l'objet d'études rigoureuses. Même s'il n'est pas essentiel de procéder à un examen neurologique complet pour le diagnostic de l'atteinte cognitive légère ou de la maladie d'Alzheimer ${ }^{3,4}$, les limites de 
l'examen neurologique virtuel posent un problème particulier pour la reconnaissance des démences associées à une maladie vasculaire cérébrale ou aux démences atypiques du spectre de la maladie de Parkinson et des syndromes parkinsoniens, qui, à l'examen, peuvent s'accompagner de déficits focaux ou de signes extrapyramidaux, respectivement ${ }^{77}$. Cela dit, certains signes classiques du Parkinson peuvent être évalués visuellement et les soins virtuels ont été utilisés pour vérifier la fonction motrice de patients atteints de Parkinson idiopathique ${ }^{78}$. De récents articles proposent la conduite à tenir pour adapter l'examen neurologique à la plateforme virtuelle, même si ces façons de procéder doivent encore être validées ${ }^{72,73}$. Au bout du compte, les médecins doivent exercer leur jugement clinique et reconnaître les situations où les limites de la consultation virtuelle justifient un retour à la consultation en personne ${ }^{14,68}$.

Finalement, pour assurer la viabilité des soins virtuels, il faut veiller à corriger les iniquités quant à leur accès. Une récente analyse transversale réalisée aux États-Unis a révélé que des caractéristiques telles que la race, l'ethnicité, la langue, le revenu et le soutien d'un proche aidant jouaient un rôle majeur dans l'accès des patients âgés aux soins virtuels par visioconférence ${ }^{79}$. Il faut mettre en place des mesures pour assurer un accès uniforme aux soins virtuels, indépendamment des facteurs sociodémographiques.

\section{Conclusion}

Les soins virtuels évoluent rapidement et leur utilisation est appelée à se répandre au fur et à mesure des avancées technologiques pour répondre à la demande des systèmes de santé. La recherche dans ce domaine s'est accélérée par suite de la pandémie de COVID-19, or, de nombreuses questions demeurent (encadré 3). Selon les publications actuelles, les outils d'évaluation cognitive administrés par soins virtuels sont généralement fiables, le déploiement d'un système de soins virtuels pour l'évaluation et la prise en charge de la maladie d'Alzheimer et des démences connexes est faisable et

Tableau 2 : Obstacles aux consultations par visioconférence directe à domicile chez les patients atteints de la maladie d'Alzheimer et de démences connexes

\section{Obstacle}

1. Accès aux technologies, littératie numérique, interférence

- Accès limité à des appareils numériques ou aux services Internet

- Littératie numérique limitée

- Réticence des patients ou des familles

- Interférence technique

\section{Alliance thérapeutique entre médecin et patient \\ - Engagement interpersonnel moindre \\ - Enjeux de protection des renseignements personnels, de confidentialité et de sécurité des données}

\section{Défis diagnostiques}

- Imprécisions résultant des conditions non standardisées de la consultation virtuelle

- Adaptation des tests cognitifs de leur version imprimée à leur version virtuelle

- Limites de l'examen neurologique à distance

\section{Pandémie de COVID-19}

- Fréquence accrue des SCPD ${ }^{10}$

- Disponibilité moindre des proches aidants pour faciliter la consultation virtuelle

\section{Solutions suggérées}

- Proposer un accès à la technologie par l'entremise de la famille, des amis ou des centres de santé locaux ${ }^{62,63}$

- Offrir des ressources, du soutien ou de la formation pour améliorer la littératie numérique des patients et des familles ${ }^{63}$

- Préparer les patients et les familles à la technologie :

- Impliquer les techniciens en télésanté26,60,64

- Envoyer les instructions à l'avance ${ }^{63,65}$

- Discuter de solutions de rechange en cas de problèmes techniques ${ }^{14,64}$

- Utiliser des stratégies verbales et non verbales pour améliorer le lien virtuel entre le médecin et le patient ${ }^{66,67}$

- Formation des professionnels de la santé en matière de bonnes pratiques en soins virtuels ${ }^{67-69}$

- Utiliser des interfaces virtuelles cryptées validées pour la protection des renseignements personnels des patients ${ }^{14,68}$

- Obtenir le consentement éclairé verbal pour les soins virtuels ${ }^{14,64}$

- Encourager le choix d'un endroit tranquille pour la consultation, où le patient peut parler seul $^{14}$

- Vérifier les déficits auditifs ou visuels et encourager l'utilisation des aides (lunettes, prothèses auditives) avant l'évaluation virtuelle $\mathrm{e}^{14,70,71}$

- Documenter les éléments à confirmer, en personne si possible ${ }^{64}$

- Demander aux patients d'imprimer à l'avance les portions écrites des tests cognitifs ${ }^{20,26}$

- Utiliser une webcam et le partage d'écran pour l'affichage 26,27,47 $^{26}$

- Adapter les tests au besoin ${ }^{26,64}$ sans modifier le construit cognitif évalué ${ }^{71}$

- Suivre les lignes directrices suggérées pour l'examen neurologique virtuel ${ }^{72,73}$

- Faire preuve de jugement si un retour à des consultations en personne s'impose $e^{14,64,68}$

- Utiliser les consultations virtuelles pour enseigner aux patients et aux proches aidants les techniques de gestion des SCPD ${ }^{51}$

- Inclure les proches aidants dans des téléconférences tripartites ${ }^{14,70}$ 


\section{Encadré 3 : Questions sans réponses}

- Les soins virtuels conviennent-ils à tout le spectre de l'atteinte cognitive et sensorielle, y compris aux patients les plus gravement atteints?

- Les soins virtuels permettent-ils d'améliorer les résultats cliniques chez les patients atteints de maladie d'Alzheimer, de démences connexes et d'autres maladies neurodégénératives?

- L'approche par soins virtuels directs à domicile est-elle faisable, acceptable et efficace pour les personnes atteintes de la maladie d'Alzheimer et de démences connexes?

- Les soins virtuels sont-ils équitablement accessibles à toutes les personnes atteintes de la maladie d'Alzheimer et de démences connexes? Sinon, quelles mesures peut-on appliquer pour assurer un accès équitable?

l'interface virtuelle semble bien acceptée. Étant donné les limites actuelles des soins virtuels, toutefois, la plupart des experts suggèrent si possible d'utiliser les consultations virtuelles en complément aux consultations en personne, plutôt qu'à leur place ${ }^{20,59}$.

La pandémie de COVID-19 a catalysé l'application à plus grande échelle des soins virtuels afin que les populations vulnérables puissent rester chez elles, en sécurité. Ultimement, si l'on veut surmonter les obstacles et faciliter l'adoption des soins virtuels pour les personnes atteintes de la maladie d'Alzheimer et de démences connexes, il sera nécessaire d'offrir aux patients, aux familles et aux professionnels de la santé plus de soutien, une bonne accessibilité, des programmes d'éducation et d'autonomisation. Il faudra des efforts de collaboration entre médecins et patients, avec l'appui des infrastructures sanitaires existantes pour réussir le déploiement des soins virtuels et favoriser l'innovation dans ce domaine.

\section{Références}

1. Shaw J, Jamieson T, Agarwal P, et al. Virtual care policy recommendations for patient-centred primary care: findings of a consensus policy dialogue using a nominal group technique. J Telemed Telecare 2018;24:608-15.

2. Gagnon M-P, Duplantie J, Fortin J-P, et al. Implementing telehealth to support medical practice in rural/remote regions: What are the conditions for success? Implement Sci 2006;1:18.

3. Albert MS, DeKosky ST, Dickson D, et al. The diagnosis of mild cognitive impairment due to Alzheimer's disease: recommendations from the National Institute on Aging-Alzheimer's Association workgroups on diagnostic guidelines for Alzheimer's disease. Alzheimers Dement 2011;7:270-9.

4. McKhann GM, Knopman DS, Chertkow H, et al. The diagnosis of dementia due to Alzheimer's disease: recommendations from the National Institute on Aging-Alzheimer's Association workgroups on diagnostic guidelines for Alzheimer's disease. Alzheimers Dement 2011;7:263-9.

5. McKeith IG, Boeve BF, Dickson DW, et al. Diagnosis and management of dementia with Lewy bodies: fourth consensus report of the DLB Consortium. Neurology 2017;89:88-100.

6. Rascovsky K, Hodges JR, Knopman D, et al. Sensitivity of revised diagnostic criteria for the behavioural variant of frontotemporal dementia. Brain 2011;134:2456-77.

7. Stephan A, Bieber A, Hopper L, et al. Barriers and facilitators to the access to and use of formal dementia care: findings of a focus group study with people with dementia, informal carers and health and social care professionals in eight European countries. BMC Geriatr 2018;18:131.

8. Bradford A, Kunik ME, Schulz P, et al. Missed and delayed diagnosis of dementia in primary care: prevalence and contributing factors. Alzheimer Dis Assoc Disord 2009;23:306.

9. Wang H, Li T, Barbarino P, et al. Dementia care during COVID-19. Lancet 2020; 395:1190-1.

10. Lara B, Carnes A, Dakterzada F, et al. Neuropsychiatric symptoms and quality of life in Spanish patients with Alzheimer's disease during the COVID-19 lockdown. Eur J Neurol 2020;27:1744-7.

11. Roach P, Zwiers A, Cox E, et al. Understanding the impact of the COVID-19 pandemic on well-being and virtual care for people living with dementia and care partners living in the community. Dementia (London) 2020 Dec. 31 [cyberpublication avant impression]. doi: 10.1177/1471301220977639.

12. Migliaccio R, Bouzigues A. Dementia and COVID-19 lockdown: more than a double blow for patients and caregivers. J Alzheimers Dis Rep 2020;4:231-5.

13. Chambers LW, Bancej C, McDowell I. Prevalence and monetary costs of dementia in Canada. Toronto: Alzheimer Society of Canada; 2016.

14. Geddes MR, O'Connell ME, Fisk JD, et al. Remote cognitive and behavioral assessment: report of the Alzheimer Society of Canada Task Force on dementia care best practices for COVID-19. Alzheimers Dement (Amst) 2020;12:e12111.

15. Hatcher-Martin JM, Adams JL, Anderson ER, et al. Telemedicine in neurology: Telemedicine Work Group of the American Academy of Neurology update. Neurology 2020;94:30-8.

16. Castanho TC, Amorim L, Zihl J, et al. Telephone-based screening tools for mild cognitive impairment and dementia in aging studies: a review of validated instruments. Front Aging Neurosci 2014;6:16.

17. Brandt J, Spencer M, Folstein M. The telephone interview for cognitive status. Neuropsychiatry Neuropsychol Behav Neurol 1988;1:111-8.

18. Cook SE, Marsiske M, McCoy KJM. The use of the Modified Telephone Interview for Cognitive Status (TICS-M) in the detection of amnestic mild cognitive impairment. J Geriatr Psychiatry Neurol 2009;22:103-9.

19. Knopman DS, Roberts RO, Geda YE, et al. Validation of the telephone interview for cognitive status-modified in subjects with normal cognition, mild cognitive impairment, or dementia. Neuroepidemiology 2010;34:34-42.

20. Frank C, John PS, Molnar F. Screening tools for virtual assessment of cognition. Can Fam Physician 2020;66:502-3.

21. Pendlebury ST, Welch SJV, Cuthbertson FC, et al. Telephone assessment of cognition after transient ischemic attack and stroke: modified telephone interview of cognitive status and telephone Montreal Cognitive Assessment versus face-to-face Montreal Cognitive Assessment and neuropsychological battery. Stroke 2013;44:227-9.

22. Carlew AR, Fatima H, Livingstone JR, et al. Cognitive assessment via telephone: a scoping review of instruments. Arch Clin Neuropsychol 2020;35: 1215-33.

23. Barth J, Nickel F, Kolominsky-Rabas PL. Diagnosis of cognitive decline and dementia in rural areas - a scoping review. Int J Geriatr Psychiatry 2018;33:459-74.

24. Brearly TW, Shura RD, Martindale SL, et al. Neuropsychological test administration by videoconference: a systematic review and meta-analysis. Neuropsychol Rev 2017;27:174-86.

25. Binng D, Splonskowski M, Jacova C. Distance assessment for detecting cognitive impairment in older adults: a systematic review of psychometric evidence. Dement Geriatr Cogn Disord 2020 Dec. 8 [cyberpublication avant impression]. doi: 10.1159/000511945.

26. Lindauer A, Seelye A, Lyons B, et al. Dementia care comes home: patient and caregiver assessment via telemedicine. Gerontologist 2017;57:e85-93.

27. liboshi K, Yoshida K, Yamaoka Y, et al. A validation study of the remotely administered Montreal Cognitive Assessment tool in the elderly Japanese population. Telemed J E Health 2020;26:920-8.

28. Loh P-K, Donaldson M, Flicker L, et al. Development of a telemedicine protocol for the diagnosis of Alzheimer's disease. J Telemed Telecare 2007;13:90-4. 
29. Cullum CM, Weiner MF, Gehrmann HR, et al. Feasibility of telecognitive assessment in dementia. Assessment 2006;13:385-90.

30. Wadsworth HE, Galusha-Glasscock JM, Womack KB, et al. Remote neuropsychological assessment in rural American Indians with and without cognitive impairment. Arch Clin Neuropsychol 2016;31:420-5.

31. Munro Cullum C, Hynan LS, Grosch M, et al. Teleneuropsychology: evidence for video teleconference-based neuropsychological assessment. J Int Neuropsychol Soc 2014;20:1028-33.

32. DeYoung N, Shenal BV. The reliability of the Montreal Cognitive Assessment using telehealth in a rural setting with veterans. J Telemed Telecare 2019;25: 197-203.

33. McEachern W, Kirk A, Morgan DG, et al. Reliability of the MMSE administered inperson and by telehealth. Can J Neurol Sci 2008;35:643-6.

34. Vestal L, Smith-Olinde L, Hicks G, et al. Efficacy of language assessment in Alzheimer's disease: comparing in-person examination and telemedicine. Clin Interv Aging 2006;1:467-71.

35. Galusha-Glasscock JM, Horton DK, Weiner MF, et al. Video teleconference administration of the repeatable battery for the assessment of neuropsychological status. Arch Clin Neuropsychol 2016;31:8-11.

36. Yoshida K, Yamaoka Y, Eguchi Y, et al. Remote neuropsychological assessment of elderly Japanese population using the Alzheimer's Disease Assessment Scale: a validation study. J Telemed Telecare 2020;26:482-7.

37. Carotenuto A, Rea R, Traini E, et al. Cognitive assessment of patients with Alzheimer's disease by telemedicine: pilot study. JMIR Ment Health 2018;5:e31.

38. Loh P-K, Ramesh P, Maher S, et al. Can patients with dementia be assessed at a distance? The use of Telehealth and standardised assessments. Intern Med J 2004;34:239-42.

39. Wadsworth HE, Dhima K, Womack KB, et al. Validity of teleneuropsychological assessment in older patients with cognitive disorders. Arch Clin Neuropsychol 2018;33:1040-5.

40. Shores MM, Ryan-Dykes P, Williams RM, et al. Identifying undiagnosed dementia in residential care veterans: comparing telemedicine to in-person clinical examination. Int J Geriatr Psychiatry 2004;19:101-8.

41. Martin-Khan M, Flicker L, Wootton R, et al. The diagnostic accuracy of telegeriatrics for the diagnosis of dementia via video conferencing. J Am Med Dir Assoc 2012;13:487.e19-24.

42. Dang S, Gomez-Orozco CA, van Zuilen MH, et al. Providing dementia consultations to veterans using clinical video telehealth: results from a clinical demonstration project. Telemed J E Health 2018;24:203-9.

43. Tso JV, Farinpour R, Chui HC, et al. A multidisciplinary model of dementia care in an underserved retirement community, made possible by telemedicine. Front Neurol 2016;7:225.

44. Powers BB, Homer MC, Morone N, et al. Creation of an interprofessional teledementia clinic for rural veterans: preliminary data. J Am Geriatr Soc 2017;65:1092-9.

45. Harrell KM, Wilkins SS, Connor MK, et al. Telemedicine and the evaluation of cognitive impairment: the additive value of neuropsychological assessment. J Am Med Dir Assoc 2014;15:600-6.

46. Weiner MF, Rossetti HC, Harrah K. Videoconference diagnosis and management of Choctaw Indian dementia patients. Alzheimers Dement 2011;7:562-6.

47. Owens AP, Ballard C, Beigi M, et al. Implementing remote memory clinics to enhance clinical care during and after COVID-19. Front Psychiatry 2020; 11:579934.

48. Powers JS, Buckner J. Reaching out to rural caregivers and veterans with dementia utilizing clinical video-telehealth. Geriatrics 2018;3:29. doi: 10.3390/ geriatrics3020029.

49. Azad N, Amos S, Milne K, et al. Telemedicine in a rural memory disorder clinic - remote management of patients with dementia. Can Geriatr J 2012;15:96.

50. Chang W, Homer M, Rossi MI. Use of clinical video telehealth as a tool for optimizing medications for rural older veterans with dementia. Geriatrics 2018;3:44. doi: 10.3390/geriatrics3030044.
51. Weiss EF, Malik R, Santos T, et al. Telehealth for the cognitively impaired older adult and their caregivers: lessons from a coordinated approach. Neurodegener Dis Manag 2021;11:83-9.

52. Possin KL, Merrilees JJ, Dulaney S, et al. Effect of collaborative dementia care via telephone and internet on quality of life, caregiver well-being, and health care use: the care ecosystem randomized clinical trial. JAMA Intern Med 2019;179:1658-67.

53. Kim H, Jhoo JH, Jang J-W. The effect of telemedicine on cognitive decline in patients with dementia. J Telemed Telecare 2017;23:149-54.

54. Cheong C-K, Lim K-H, Jang J-W, et al. The effect of telemedicine on the duration of treatment in dementia patients. J Telemed Telecare 2015;21:214-8.

55. Lyketsos CG, Roques $C$, Hovanec $L$, et al. Telemedicine use and the reduction of psychiatric admissions from a long-term care facility. J Geriatr Psychiatry Neurol 2001;14:76-9.

56. Lee $\mathrm{JH}$, Kim JH, Jhoo JH, et al. A telemedicine system as a care modality for dementia patients in Korea. Alzheimer Dis Assoc Disord 2000;14:94-101.

57. Parikh M, Grosch MC, Graham LL, et al. Consumer acceptability of brief videoconference-based neuropsychological assessment in older individuals with and without cognitive impairment. Clin Neuropsychol 2013;27:808-17.

58. Morgan DG, Kosteniuk J, Stewart N, et al. The telehealth satisfaction scale: reliability, validity, and satisfaction with telehealth in a rural memory clinic population. Telemed J E Health 2014;20:997-1003.

59. Hantke NC, Gould C. Examining older adult cognitive status in the time of COVID-19. J Am Geriatr Soc 2020;68:1387-9.

60. Moo LR, Gately ME, Jafri Z, et al. Home-based video telemedicine for dementia management. Clin Gerontol 2020;43:193-203.

61. Benaque A, Gurruchaga MJ, Abdelnour C, et al. Dementia care in times of COVID-19: Experience at Fundació ACE in Barcelona, Spain. J Alzheimers Dis 2020;76:33-40.

62. Tousi B. Dementia care in the time of COVID-19 pandemic. J Alzheimers Dis 2020;76:475-9.

63. Parker CB, Forbes MP, Vahia IV, et al. Facing the change together: reflections of coping and resilience from american geriatric psychiatrists during COVID-19. Am J Geriatr Psychiatry 2020;28:1015-9.

64. Hewitt KC, Loring DW. Emory university telehealth neuropsychology development and implementation in response to the COVID-19 pandemic. Clin Neuropsychol 2020;34:1352-66.

65. Walsh J, Markus HS. Telemedicine for follow-up of rare neurological disease Stroke 2019;50:750-3.

66. Bulik RJ. Human factors in primary care telemedicine encounters. J Telemed Telecare 2008;14:169-72.

67. Liu X, Sawada Y, Takizawa T, et al. Doctor-patient communication: a com parison between telemedicine consultation and face-to-face consultation. Intern Med 2007;46:227-32.

68. Wechsler LR. Advantages and limitations of teleneurology. JAMA Neurol 2015;72:349-54

69. Terry C, Cain J. The emerging issue of digital empathy. Am J Pharm Educ 2016;80:58.

70. Steinman MA, Perry L, Perissinotto CM. Meeting the care needs of older adults isolated at home during the COVID-19 pandemic. JAMA Intern Med 2020;180:819-20.

71. Phillips NA, Chertkow H, Pichora-Fuller MK, et al. Special issues on using the Montreal Cognitive Assessment for telemedicine assessment during COVID-19. J Am Geriatr Soc 2020;68:942-4.

72. Al Hussona M, Maher M, Chan D, et al. The Virtual Neurologic Exam: instructional videos and guidance for the COVID-19 era. Can J Neurol Sci 2020;47:598-603.

73. Boes CJ, Hunderfund ANL, Martinez-Thompson JM, et al. A primer on the in-home teleneurologic examination: a COVID-19 pandemic imperative. Neurol Clin Pract 2020. doi: 10.1212/CPJ.0000000000000876. Accessible ici : https://cp.neurology.org/content/early/2020/05/21/CPJ.0000000000000876. abstract (consulté le 20 juill. 2020). 
74. Lam K, Lu AD, Shi Y, et al. Assessing telemedicine unreadiness among older adults in the United States during the COVID-19 pandemic. JAMA Intern Med 2020;180:1389-91.

75. Rosenberg L, Kottorp A, Winblad B, et al. Perceived difficulty in everyday technology use among older adults with or without cognitive deficits. Scand $J$ Occup Ther 2009;16:216-26.

76. Moyle W, Jones C, Murfield J, et al. "For me at 90, it's going to be difficult": feasibility of using iPad video-conferencing with older adults in long-term aged care. Aging Ment Health 2020;24:349-52.
77. Knopman DS, Boeve BF, Petersen RC. Essentials of the proper diagnoses of mild cognitive impairment, dementia, and major subtypes of dementia. Mayo Clin Proc 2003;78:1290-308.

78. Adams JL, Myers TL, Waddell EM, et al. Telemedicine: a valuable tool in neurodegenerative diseases. Curr Geriatr Rep 2020;9:72-81.

79. Schifeling $\mathrm{CH}$, Shanbhag $\mathrm{P}$, Johnson $\mathrm{A}$, et al. Disparities in video and telephone visits among older adults during the COVID-19 pandemic: cross-sectional analysis. JMIR Aging 2020;3:e23176.
Intérêts concurrents : Mario Masselis déclare avoir reçu des subventions indépendamment des travaux soumis ici de la part des Instituts de recherche en santé du Canada, du Ministère du Développement économique et de la Croissance de l'Ontario, de l'Institut ontarien du cerveau, du Sunnybrook AFP Innovation Fund, de l'Alzheimer's Drug Discovery Foundation, de la Fondation Brain Canada, du Stroke Foundation Centre for Stroke Recovery, du Weston Brain Institute, de Roche, de I'Université de Washington, d'Axovant et d'Alector. Il signale aussi avoir reçu des honoraires personnels indépendamment des travaux soumis ici de la part d'Arkuda Therapeutics, d'lonis, d'Henry Stewart Talks, d'Alector, de Biogen Canada et de Wave Life Sciences. Il est également co-rédacteur pour Current Pharmacogenomics and Personalized Medicine. Aucun autre intérêt concurrent déclaré.

Cet article a été révisé par des pairs.

Affiliations : Faculté de médecine (Gosse, Kassardjian, Masellis, Mitchell) et Département de médecine, Division de neurologie (Kassardjian, Masellis, Mitchell), Université de Toronto; Centre des sciences de la santé Sunnybrooke, Service de médecine, Division de neurologie (Masellis, Mitchell); Service de médecine, Division de neurologie (Kassardjian), Hôpital St. Michael; Neurology Quality and Innovation Lab (NQIL) (Kassardjian, Mitchell), Université de Toronto; Hurvitz Brain Sciences Research Program (Masellis, Mitchell), Institut de recherche Sunnybrook; Institut du savoir Li Ka Shing (Kassardjian), Hôpital St. Michael, Toronto, Ont.
Collaborateurs : Tous les auteurs ont contribué à l'élaboration et à la conception des travaux ainsi qu'à l'acquisition, à l'analyse et à l'interprétation des données. Tous les auteurs ont participé à la rédaction du manuscrit, en ont révisé de façon critique le contenu intellectuel important, ont donné leur approbation finale pour la version destinée à être publiée, et l'entière responsabilité de tous les aspects du travail.

Financement : Mario Masellis a reçu du financement de l'Ontario Neurodegenerative Disease Research Initiative (ONDRI) pour la recherche, la rédaction et la publication de cet article. L'ONDRI reçoit des subventions de recherche de l'Ontario Brain Institute, une corporation sans but lucratif indépendante, financée en partie par le gouvernement de l'Ontario.

Avis : Les opinions, les résultats et les conclusions n'engagent que les auteurs et aucun appui de la part de l'Ontario Brain Institute n'est sousentendu ni devrait être inféré.

Propriété intellectuelle du contenu : Il s'agit d'un article en libre accès distribué conformément aux modalités de la licence Creative Commons Attributions (CC BY-NC-ND 4.0), qui permet l'utilisation, la diffusion et la reproduction dans tout médium à la condition que la publication originale soit adéquatement citée, que l'utilisation se fasse à des fins non commerciales (c.-à-d. recherche ou éducation) et qu'aucune modification ni adaptation n'y soit apportée. Voir : https://creativecommons. org/licenses/by-nc-nd/4.0/deed.fr.

Correspondance : Sara Mitchell, sara.mitchell@sunnybrook.ca 